OPEN ACCESS

Edited by:

Joyce Van Eck,

Boyce Thompson Institute,

United States

Reviewed by:

Xiangbing Meng,

Institute of Genetics and

Developmental Biology (CAS), China

Jongjin Park,

Naturegenic, United States

*Correspondence:

Wenzhi Jiang

jiang@molbio.mgh.harvard.edu

Specialty section:

This article was submitted to

Genome Editing in Plants,

a section of the journal

Frontiers in Genome Editing

Received: 02 June 2021 Accepted: 30 November 2021

Published: 22 December 2021

Citation:

Jiang W, Bush J and Sheen J (2021) A Versatile and Efficient Plant Protoplast

Platform for Genome Editing by

Cas9 RNPs.

Front. Genome Ed. 3:719190.

doi: 10.3389/fgeed.2021.719190

\section{A Versatile and Efficient Plant Protoplast Platform for Genome Editing by Cas9 RNPs}

\author{
Wenzhi Jiang *, Jenifer Bush and Jen Sheen
}

Department of Molecular Biology and Center for Computational and Integrative Biology, Massachusetts General Hospital, and Department of Genetics, Harvard Medical School, Boston, MA, United States

The ultimate goal of technology development in genome editing is to enable precisely targeted genomic changes in any cells or organisms. Here we describe protoplast systems for precise and efficient DNA sequence changes with preassembled Cas9 ribonucleoprotein (RNP) complexes in Arabidopsis thaliana. Nicotiana benthamiana, Brassica rapa, and Camelina sativa. Cas9 RNP-mediated gene disruption with dual gRNAs could reach $\sim 90 \%$ indels in Arabidopsis protoplasts. To facilitate facile testing of any Cas9 RNP designs, we developed two GFP reporter genes, which led to sensitive detection of nonhomologous end joining (NHEJ) and homology-directed repair (HDR), with editing efficiency up to 85 and 50\%, respectively. When co-transfected with an optimal single-stranded oligodeoxynucleotide (ssODN) donor, precise editing of the AtALS gene via HDR reached $7 \%$ by RNPs. Significantly, precise mutagenesis mediated by preassembled primer editor (PE) RNPs led to 50\% GFP reporter gene recovery in protoplasts and up to $4.6 \%$ editing frequency for the specific AtPDS mutation in the genome. The rapid, versatile and efficient gene editing by CRISPR RNP variants in protoplasts provides a valuable platform for development, evaluation and optimization of new designs and tools in gene and genomic manipulation and is applicable in diverse plant species.

Keywords: plant protoplasts, Cas9 RNP, NHEJ, HDR, ssODN donor, prime editor RNP, Arabidopsis thaliana, Nicotiana benthamiana

\section{INTRODUCTION}

Successful CRISPR-Cas9-gRNA-mediated genome editing in plant cells was first demonstrated in protoplasts isolated from Arabidopsis, tobacco, rice and wheat with varying degrees of efficiency for various targets in different plants (Li et al., 2013; Shan et al., 2013). Despite widespread applications of CRISPR technologies via NHEJ-mediated mutagenesis in basic plant research and crop improvement (Zhu et al., 2020), the underlying mechanisms for variable editing efficiency and limited precise editing through HDR remain unresolved challenges for future advances (Chechik et al., 2020; Zhang et al., 2021). Recent CRISPR-Cas9 innovations have significantly expanded the range of genetic variants by PEs (Anzalone et al., 2019; Lin et al., 2020, Jin et al., 2021) and enabled targeted insertion and replacement (Lu et al., 2020). However, the DNA- and transgene-based approaches using Agrobacterium- and particle bombardment-mediated plant transformation still face many restrictions (Lin et al., 2020; Lu et al., 2020). The emerging and flexible CRISPR RNPs may offer multiple advantages in DNA-, cloning- and transgene-free genome editing with higher 
efficiency and precision, minimal off targets, and reduced toxicity. Furthermore, the versatile plant protoplast platform with high transfection efficiency (Yoo et al., 2007; Li et al., 2013, 2014, 2015; Marx, 2016) is highly suitable in supporting the systematic efforts required for developing and testing new CRISPR-Cas9 designs and elucidating relevant molecular mechanisms in broad plant species (Woo et al., 2015; Jin et al., 2021; Zhang et al., 2021).

In this study, we integrated Cas9 RNPs with highly efficient protoplast transfection systems for rapid analyses of gene and genomic editing efficiency. Two reporter genes were developed to facilitate sensitive detection of NHEJ or HDR leading to the recovery of GFP fluorescence in transfected protoplasts within $24 \mathrm{~h}$. We demonstrated that this versatile protoplast platform could be applied to four plant species and support precise and efficient mutagenesis by HDR using relatively short ssODN donors without costly modifications. When combined with a specific ssODN and dual gRNAs, Cas9 RNPs enabled the generation of a precise mutation in the AtALS gene, not previously feasible in Arabidopsis protoplasts featuring low editing efficiency with plasmid DNA despite high cell quality and transfection efficiency (Yoo et al., 2007; Li et al., 2013). Importantly, we provided the first evidence that precise mutations free of unintended indels (Jin et al., 2021) could be efficiently generated by PE-mediated editing in the GFP reporter and in the Arabidopsis genome using the preassembled nCas9-RT RNP complexes without double-strand break, donor DNA or the additional DNA nick. This versatile and efficient protoplast platform will help enable plant researchers aiming to test novel Cas9-gRNA variants or create new CRISPR designs and tools for facile genome manipulation in model or crop plants.

\section{MATERIALS AND METHODS}

\section{Plant Materials and Growth Conditions}

Seeds of wild-type Arabidopsis thaliana (A. thaliana) Col-0, tobacco Nicotiana benthamiana (N. benthamiana), Brassica rapa ( $B$. rapa) and Camelina sativa ( $C$. sativa) were germinated and grown on Jiffy-7 peat pellets (Jiffy group) except that $N$. benthamiana seedlings (2 weeks) were transferred to ProMix BK25 soil (Premier Tech Horticulture) in a controlled environment growth room at $12 \mathrm{~h} \mathrm{light,} 23^{\circ} \mathrm{C} / 12 \mathrm{~h}$ dark, $20^{\circ} \mathrm{C}$ under low light $\left(75 \mu \mathrm{E} \mathrm{m}^{-2} \mathrm{~s}^{-1}\right)$ and $60 \%$ relative humidity. N. benthamiana plants were fertilized with $1 / 4 \mathrm{X}$ Hoagland solution once per week, beginning at 3 weeks. Vigorously growing and well-expanded leaves from 24 to 32 days (leaf 1-6) were chosen for mesophyll protoplast isolation. Optimal leaves were systematically tested and selected for high quality mesophyll protoplast isolation with high transfection efficiency. For example, we used leaf $5 \& 6$ at 30 days for $A$. thaliana, leaf $5 \& 6$ at 32 days for $N$. benthamiana, leaf $1 \& 2$ at 24 days for B. rapa and leaf $3 \& 4$ at 29 days for $C$. sativa. Older leaves or plants recovered from stress conditions yielded low quality protoplasts with low transfection efficiency, hence low gene and genome editing efficiency (Yoo et al., 2007; Li et al., 2013, 2014, 2015).

\section{Plasmid Construction}

For constructing the NHEJ GFP reporter gene (NHEJ-GFP), the DOF1-GFP gene (Yanagisawa and Sheen (1998) driven by the AtUBQ10 promoter with the NOS terminator in a pUC19 cloning vector was used to initiate the design. We inserted a 20-bp target sequence plus a PAM sequence (AGG) (GCGCTTCAAGGTGCA CATGGAGG) at the 5' end of the GFP gene, which led to an outof-frame shift in the coding region. A double-strand cleavage within this target region by Cas9-gRNA will generate some indels leading to in-frame shifts in the mutated GFP coding region allowing the recovery of the GFP fluorescence after NHEJmediated DNA repair in transfected protoplasts (Figure 3A). For the construction of the HDR GFP reporter gene (HDR-GFP), the chromophore 65TYG67 (Chiu et al., 1996) was mutated to 65TLR67 (nucleotide sequence from ACC TAC GGC to ACC TTA CGC) to inactivate GFP. Only a precise DNA sequence editing by Cas 9 or PE RNPs, in which the mutation is converted back to its original chromophore 65TYG67 from 65TLR67 (from ACC TTA CGC to ACC TAC GGC) can recover the GFP fluorescence in protoplasts (Figure 3B). Full coding region sequences of the NHEJ-GFP and HDR-GFP reporter genes are listed in Supplementary Materials.

To construct Streptococcus pyogenes Cas9 protein expression plasmid, the codon optimized Cas 9 sequence for E. coli expression with a monopartite nuclear localization signal (NLS) (PKKKRKV) at the C terminus with 6XHIS tag in the pET15 expression vector (D’Astolfo et al., 2015) was fused to a bipartite NLS (KRPAATKKAGQAKKKK) at the $\mathrm{N}$ terminus to generate $b N L S C a s 9$. For constructing the plasmid to express $\mathrm{PE}$ in E. coli, we mutated $b N L S C a s 9$ to a nickase (H840A) and fused the engineered M-MLV reverse transcriptase (RT) and the linker from PE2 (Anzalone et al., 2019) to the C terminus to generate $n$ Cas $9-R T$. The coding sequences of bNLSCas9 and $n$ Cas $9-R T$ plasmids are listed in Supplementary Materials.

\section{Protein Expression and Purification}

The bNLSCas 9 or $n$ Cas $9-R T$ plasmid was transformed into E. coli BL21 (DE3) cells and single colony was picked and grown overnight at $37^{\circ} \mathrm{C}$ in $5 \mathrm{ml}$ Terrific Broth medium. This $5 \mathrm{ml}$ culture was further propagated in $2 \mathrm{~L}$ flask containing $500 \mathrm{ml}$ of LB medium and grown at $220 \mathrm{rpm}$ in a $37^{\circ} \mathrm{C}$ shaker. When cell density reached $\mathrm{OD}_{600}=0.5$ after $3-4 \mathrm{~h}$, the culture was transferred into $180 \mathrm{rpm}$ and $30^{\circ} \mathrm{C}$ for another $30 \mathrm{~min}$ before adding $1 \mathrm{mM}$ IPTG to induce protein expression. The culture was kept at $180 \mathrm{rpm}$ and $18^{\circ} \mathrm{C}$ for $48 \mathrm{~h}$. Cells were then harvested and the pellet was re-suspended in $10 \mathrm{ml}$ buffer, $50 \mathrm{mM} \mathrm{NaH}_{2} \mathrm{PO}_{4} \mathrm{pH}$ $8,1 \mathrm{M} \mathrm{NaCl}, 10 \mathrm{mM}$ 2-mercaptoethanol and $2.5 \mathrm{mM} \mathrm{MgCl}_{2}$ and protease inhibitors (cOmplete, EDTA-free, Roche 05056489001). The $10 \mathrm{ml}$ sample was then sonicated for $6 \mathrm{~min}\left(10^{\prime \prime}\right.$ on and $10^{\prime \prime}$ off, 30\% amplitude) on ice using a Q700 sonicator (Qsonica). After sonication, the sample was spun down for $30 \mathrm{~min}$ at $20,000 \times \mathrm{g}$, and $4^{\circ} \mathrm{C}$. The supernatant was collected and applied onto a $35 \mathrm{ml}$ column filled with $1 \mathrm{ml}$ High Affinity NickelCharged resin (GenScript L00223). The sample was washed twice with the re-suspension buffer. Proteins were eluted from column by using a re-suspension buffer containing 200-250 mM imidazole. Elutes were pooled together and concentrated to 
$\sim 5 \mu \mathrm{g} / \mu \mathrm{l}$ by Amicon Ultra- 15 with $10 \mathrm{KDa}$ cutoff. The buffer for concentrated protein was exchanged by dialysis using 3500 MWCO cassette (Slide-A-Lyzer 3.5K Dialysis Cassettes, Thermo Scientific 66,330) at $4^{\circ} \mathrm{C}$ for $16 \mathrm{~h}$. The storage buffer contains $20 \mathrm{mM}$ HEPES pH 7.5, $150 \mathrm{mM} \mathrm{KCI,} 2 \mathrm{mM}$ DTT and $10 \%$ glycerol. Purity of proteins was examined by protein gel electrophoresis and staining before storage at $-80^{\circ} \mathrm{C}$.

\section{Design of gRNA and pegRNA and in Vitro Synthesis}

Design of gRNAs and pegRNAs followed the methods described previously (Li et al., 2013, 2015; Anzalone et al., 2019; Jin et al., 2021). For in vitro synthesis of the gRNA or pegRNA targeting the GFP reporter gene or different sites in the genome, DNA from a plasmid containing the sequence of full-length scaffold gRNA was used as the common template, and a full-length DNA template was PCR amplified using a pair of designed forward and reverse primers (see Supplementary Tables S1, S2, S4). In vitro gRNA synthesis was undertaken using RNA synthesis kit HiScribe ${ }^{\mathrm{TM}} \mathrm{T} 7$ Quick High Yield RNA Synthesis (NEB) and purified by RNeasy Mini Kit (Qiagen).

\section{Analysis of Cas9 RNP Activity in Vitro}

Cas9 RNP activities were determined in vitro using $20 \mu \mathrm{l}$ reactions containing $2 \mu \mathrm{l}$ NEB CutSmart Buffer, $1 \mu \mathrm{g}$ Cas9 protein, $1 \mu \mathrm{g}$ gRNA for preassembly at $23^{\circ} \mathrm{C}$ for $10 \mathrm{~min}$. Linearized plasmid DNA $(0.2 \mu \mathrm{g}$ ) with the designed AtBON1 (At5G61900) target site (Li et al., 2015) was then added and incubated at $37^{\circ} \mathrm{C}$ for $30 \mathrm{~min}$ before visualization by running a $2 \%$ agarose gel and staining with ethidium bromide for UV imaging.

\section{Preassembly of Cas9 RNPs}

For preassembly of Cas9 RNPs, $2 \mu \mathrm{l} \mathrm{NEB} \mathrm{10X} \mathrm{Buffer} \mathrm{3,} 20 \mu \mathrm{g}$ gRNA or pegRNA and $40 \mu \mathrm{g}$ of bNLSCas 9 protein or nCas9-RT protein was mixed to a final volume of $20 \mu$ l. The preassembly of Cas9 RNPs was performed at $30^{\circ} \mathrm{C}$ for $30 \mathrm{~min}$ without visible precipitation in the mix.

\section{Protoplast Isolation and Transfection}

Methods for mesophyll protoplast isolation from leaves and transfection with optimal concentration of plasmid DNA to express Cas 9 and gRNA were described previously (Yoo et al., 2007; Li et al., 2013; Li et al., 2015) with minor modifications. The analyses of GFP reporter editing and genome editing were conducted after dark incubation of transfected protoplasts at $24 \mathrm{~h}$ instead of $36 \mathrm{~h}$ based on extensive comparison of prior and current results (Li et al., 2013; Li et al., 2015). Longer incubation of transfected protoplasts beyond $24 \mathrm{~h}$ did not significantly increase the editing efficiency with both plasmid DNA or RNPs. Specific plant age and leaf number for four plant species are described in plant materials and growth conditions and in the Results. Plasmid DNA or ssODN was added to protoplasts and mixed well before adding Cas RNPs for PEGcalcium-mediated transfection. In the case when preassembled Cas9 RNPs were used, protoplasts were incubated at $30^{\circ} \mathrm{C}$ for $20 \mathrm{~min}$ after the PEG-calcium treatment. Protoplasts were washed twice using W5 buffer before incubation in WI buffer.
Typically, $100 \mu \mathrm{l}$ cells at $3 \times 10^{5} \mathrm{ml}^{-1}, 15 \mu \mathrm{g}$ GFP reporter plasmid DNA, $30 \mu \mathrm{g}$ ssODN, $20 \mu \mathrm{g}$ gRNA or pegRNA, and $40 \mu \mathrm{g}$ Cas 9 protein were used in each transfection.

\section{Editing Efficiency Evaluation by GFP Imaging in Protoplasts}

Evaluation of editing efficiency for NHEJ or HDR mediated by Cas9-gRNA expressed from transfected plasmid DNA or Cas9RNPs was quantified by counting the percentage recovery of GFP in plant protoplasts. UBQ10-NLS-tdTomato (Emonet et al., 2021) was co-transfected and served as a control to mark the transfected protoplasts and determine the transfection efficiency. Percentage recovery of the GFP reporter gene in protoplasts was quantified by fluorescence microscopy as a ratio of cells with visible GFP signal to 100 total transfected cells expressing nuclear tdTomato.

\section{Editing Efficiency Evaluation in the Genome} Protoplasts were harvested $24 \mathrm{~h}$ after transfection by pelleting protoplasts, resuspending the cells in $20 \mu \mathrm{l} \mathrm{TE}$ buffer and boiling at $95^{\circ} \mathrm{C}$ for $10 \mathrm{~min}$. PCR amplification of target regions of 200-240 bp spanning individual Cas9-gRNA target sequences was performed using Phusion high-fidelity DNA polymerase (NEB) with $2 \mu$ protoplast lysates (see Supplementary Table S3, S5 and S6 for primers used for PCR). PCR amplicons (200-240 bp) were analyzed by next-generation sequencing (NGS) paired-end reads yielding typically up to 80,000 reads for each sample. The percentage of WT and each variant sequence was determined in order to estimate the editing efficiency. The amplicon NGS data were submitted to NCBI (PRJNA781623) and presented in Supplementary Figure S5.

For the analysis of off-target frequency, off-target sites were predicted using Cas-OFFinder tool (www.rgenome.net). Primers for PCR amplifying target regions are listed in Supplementary Table S6. PCR amplicons were analyzed by NGS described as above.

\section{RESULTS}

\section{Optimal Leaf Selection for Protoplast Isolation and Transfection}

The quality of mesophyll protoplasts isolated from plant leaves is critical for highly efficient transfection of DNA, RNA and proteins, and is essential to the level of gene expression and gene editing efficiency by Cas9-gRNA (Yoo et al., 2007; Li et al., 2013). Environmental factors, such as light, photoperiod, temperature, water, nutrients, soil, and relative humidity, are key to support healthy plant growth and determine the developmental timing and stage of each leaf. The physiological conditions and the age of the leaves strongly influence the quality of isolated protoplasts. Four different plant species, A. thaliana, $N$. benthamiana, B. rapa and C. sativa, were used in this study to illustrate the selection of optimal leaves at a specific age grown in a defined environmental condition (Figure 1A). For example, young and actively expanding leaf 5 and leaf 6 , but not older leaf 3 , from the same 30 -day-old Arabidopsis plants grown in a defined environment (details in MATERIALS AND 
A
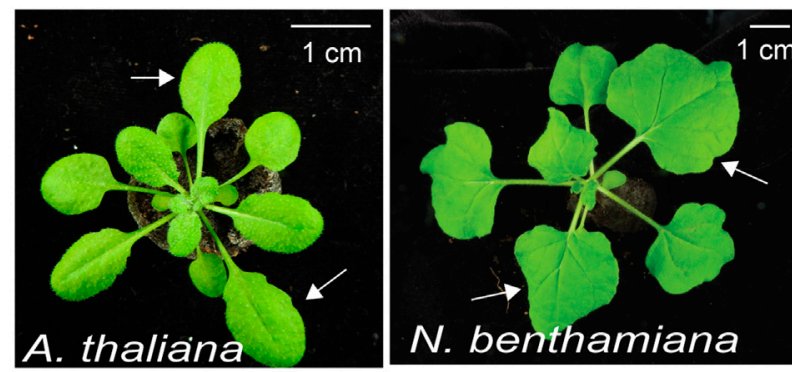
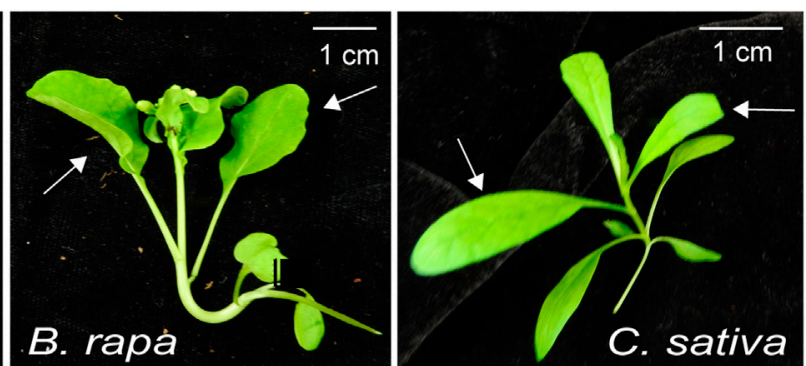

B

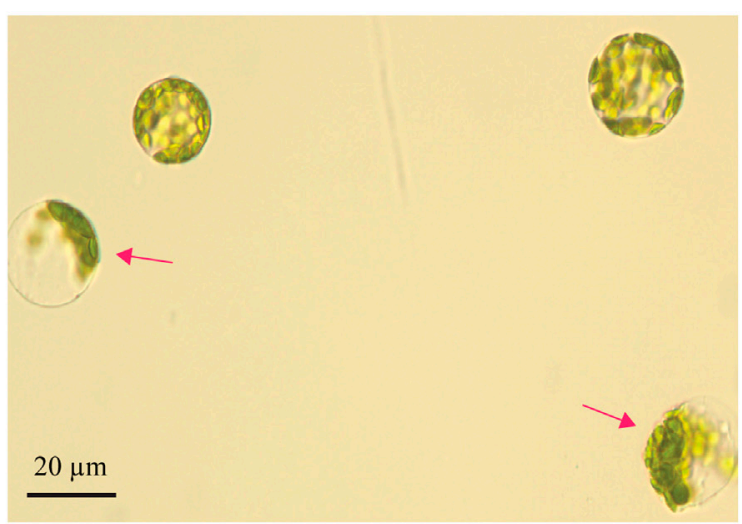

A.t. Protoplasts from leaf 3

C

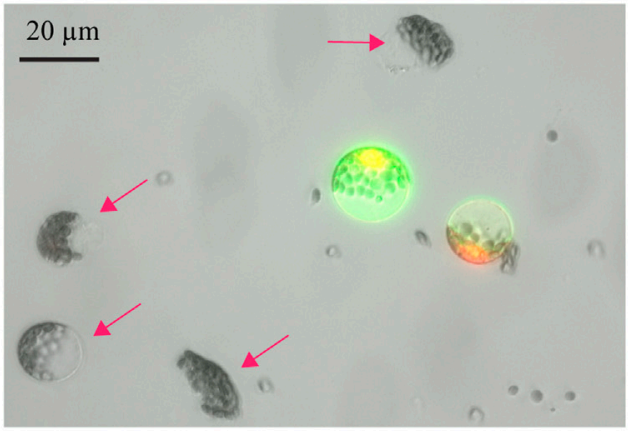

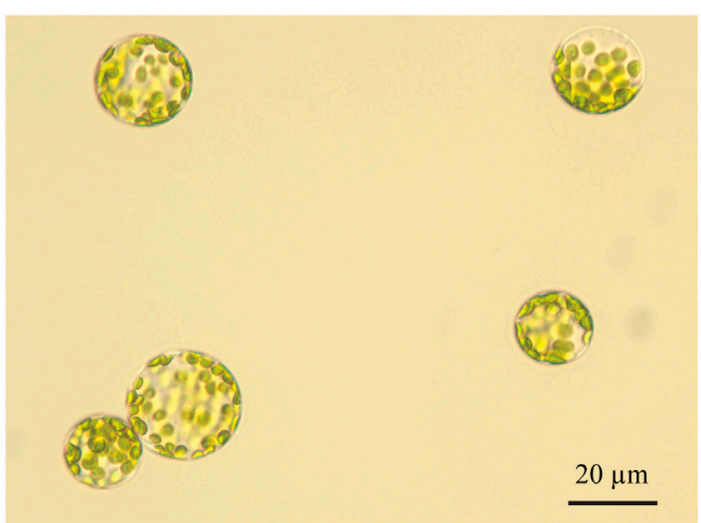

A.t. Protoplasts from leaf 5

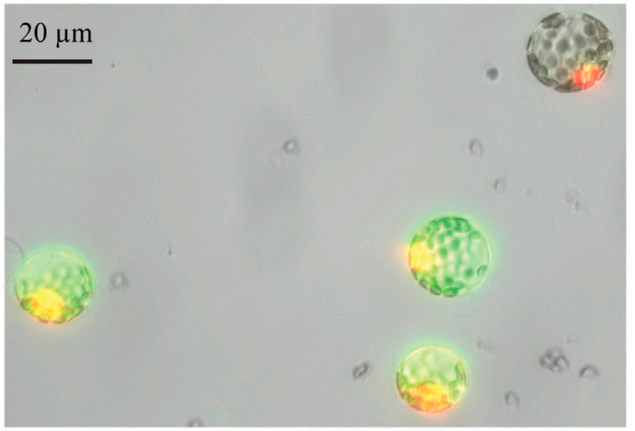

Transfected A.t. protoplasts from leaf 3 Transfected A.t. protoplasts from leaf 5

FIGURE 1 | Optimal leaf selection for the mesophyll protoplast platform. (A) Representative leaves for high quality protoplast isolation with high transfection efficiency. A. thaliana (leaf $5 \& 6$ at 30 days), N. benthamiana (leaf $5 \& 6$ at 32 days), B. rapa (leaf 1 \& 2 at 24 days) and C. sativa (leaf 3 \& 4 at 29 days). (B) Integrity of protoplasts isolated from older leaf 3 and younger leaf 5 in the same $A$. thaliana plants. (C) Transfection efficiency and integrity of protoplasts isolated from older leaf 3 and younger leaf 5. Transfected protoplasts show GFP fluorescence. Red arrows indicate low quality protoplasts from older leaf 3 . Reproducible results were obtained from at least two independent biological experiments.

METHODS) provided active protoplasts for gene expression based on numerous studies (Yoo et al., 2007; Li et al., 2013). As shown in Figure 1B, many protoplasts isolated from older leaf 3 exhibited compromised integrity but not protoplasts isolated from younger leaf 5 (leaf 3 integrity of $60.1 \%, \pm 7.1$ vs. leaf 5 integrity of $93.0 \%, \pm 2.6 ; n=3, p<0.01$ ). Transfection efficiency of protoplasts isolated from older leaf 3 was also much lower based on the expression of the GFP reporter (leaf 3 transfection efficiency of $42.5 \%, \pm 1.26$ vs. leaf 5 transfection efficiency of
$90.0 \%, \pm 0.88 ; n=3, p<0.001$ ) (Figure 1C). It was clear that comparing leaves at different developmental ages in the same plants for protoplast integrity and transfection efficiency identified the optimal leaves for the most robust mesophyll protoplasts suitable for transient expression experiments. Using a similar growth condition and protoplast tests, leaf $5 \&$ 6 at 32 days, leaf $1 \& 2$ at 24 days, and leaf $3 \& 4$ at 29 days were optimal for protoplast isolation in N. benthamiana, B. rapa and $C$. sativa, respectively (Figure 1A). 


\section{A AtBON 1 (AT5G61900)}

\section{8 bp AtBON 1}

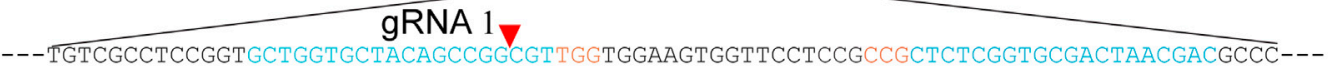

---ACAGCGGAGGCCACGACCACGATGTCGGCCGCAACCACCTTCACCAAGGAGGCGGCGAGAGCCACGCTGATTGCTGCGGG---

B
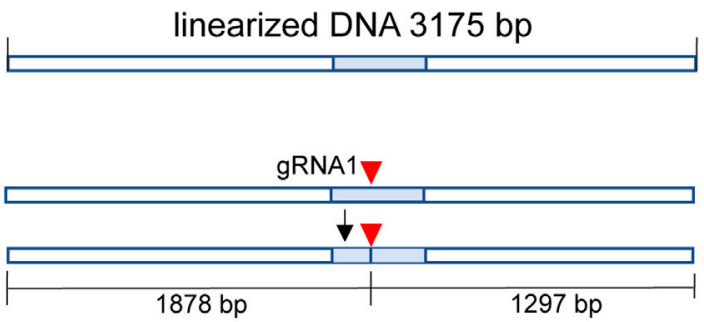

\section{C}
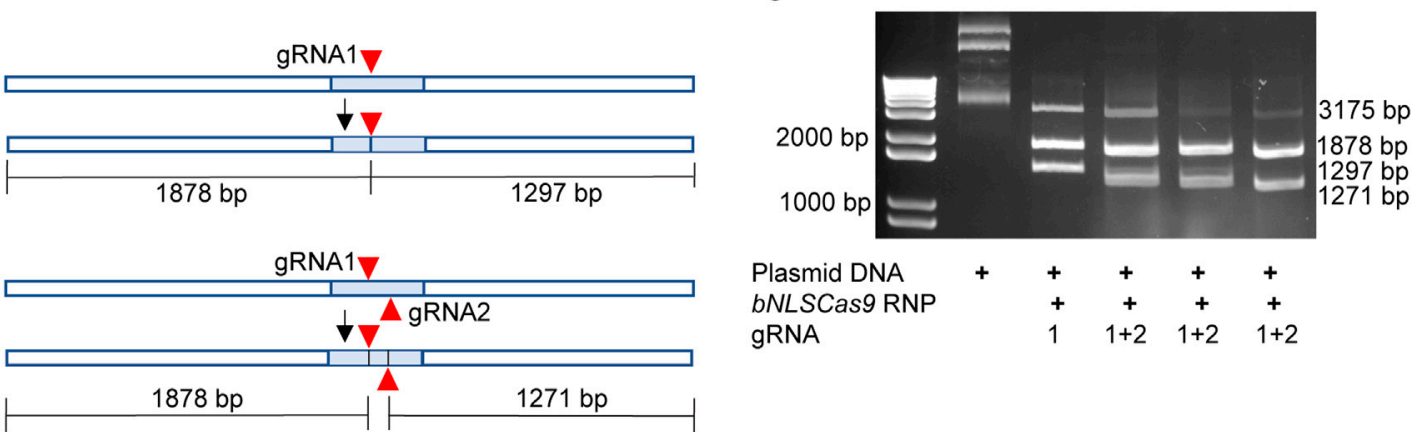

D

AtBON 1 gRNA 1

AtBON 1 gRNA 2

RNP TCCGGTGCTGGTGCTACAGCCGGCGTTGGTGGAAGTGGTTCCTCCGCCGCTCTCGGTGCGACTAACGACGCCCTTG WT

Exp. 1 TCCGGTGCTGGTGCTACAGCCGG-92.4\% TCCGGTGCTGGTGCTACAGCCGGCGTTGGTGGAAGTGGTTCCTCCGCCGCTCTCGGTGCGACTAACGACGCCCTTG $7.59 \%$ TCCGGTGCTGGTGCTACAGCCGG---TCCGGTGCTGGTGCTACAGCCGGCG_-_._._. TCCGGTGCTGGTGCTACAGCCGG-.-. TCCGGTGCTGGTGCTACAGCCG-_-_. TCCGGTGCTGGTGCT $--\ldots$ TCCGGTGCTGGTGCTACAGCCGG-_._. TCCGGTGCTGGTGCTACAGCCGG-..-_... TCCGGTGCTGGTGCTACAGCCGG---_-n-

DNA TCCGGTGCTGGTGCTACAGCCGGCGTTGGTGGAAGTGGTTCCTCCGCCGCTCTCGGTGCGACTAACGACGCCCTTG WT

Exp. 1 TCCGGTGCTGGTGCTACAGCCGGCGTTGGTGGAAGTGGTTCCTCCGCCGCTCTCGGTGCGACTAACGACGCCCTTG $82.4 \%$

$17.6 \%$ TCCGGTGCTGGTGCTACAGCCGG--TCCGGTGCTGGTGCTACAGCCGG---TCCGGTGCTGGTGCTACAGCCGGCAGTTGGTGGAAGTGGTTCCTCCGCCGCTCTCGGTGCGACTAACGACGCCCTTG $0.67 \%$ TCCGGTGCTGGTGCTACAGCCGG---

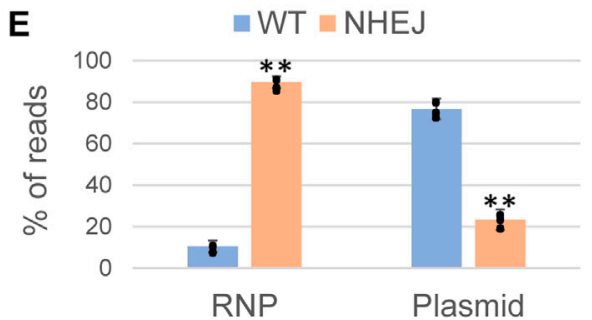

FIGURE 2 |Analyses of bNLSCas9 RNP in vitro and in protoplasts. (A) Schematic presentation of the linearized plasmid carrying the AtBON 1 editing target region. The sequence of $80 \mathrm{bp}$ with two gRNAs is shown with the PAM sequence in red. (B) Schematic presentation of in vitro AtBON 1 editing. Red arrowheads indicate the double-strand break sites. (C) bNLSCas9 RNP-mediated double-strand break at AtBON 1 in vitro and predicted plasmid DNA fragments are shown. (D) Comparison of AtBON 1 editing via NHEJ by bNLSCas9 RNP or DNA transfection in Arabidopsis protoplasts. Indel (red) \% of one representative biological experiment is shown by NGS analyses of the amplicons generated by PCR using genomic DNA isolated from transfected protoplasts. (E) The AtBON1 editing efficiency from three biological repeats $\left({ }^{\star \star} p<0.01\right.$; error bars, s.d., $n=3$ ). 


\section{Validation of Cas9-RNP Activity in Vitro and in Vivo}

Abundant bNLSCas9 protein was successfully overexpressed in E. coli, purified by one-step high affinity Nickel-charged resin (D'Astolfo et al., 2015), and tested for in vitro and in vivo activities using the previously validated dual gRNA target sites in the AtBON1 gene (AT5G61900) (Figure 2A) (Li et al., 2015). For the in vitro assay, the 3,175 bp plasmid DNA carrying the 238-bp insert from $A t B O N 1$ was linearized and subjected to doublestrand cleavage by preassembled Cas9-RNPs with one or two gRNAs (Figure 2B). The results showed that Cas9-RNPs generated the predicted 1878-bp and 1297-bp DNA fragments with gRNA1, which was coupled with the significant reduction of the 3175-bp linear plasmid DNA. Two gRNAs supported even higher cleavage efficiency (Figure 2C). In Arabidopsis protoplasts transfected with Cas9-RNPs preassembled with dual gRNAs targeting the ATBON1 gene, NGS analyses revealed a remarkably high editing efficiency up to $92.4,89.5$ and $86.7 \%$ in three biologically independent experiments. There was 3.8 -fold increase when the editing efficiency was compared between Cas9RNPs and Cas9-gRNA expression by conventional plasmid DNA transfection (Figure 2D, Supplementary Figure S1), consistent with the previously reported $20 \%$ editing efficiency with dual gRNAs in AtBON1 by DNA transfection in Arabidopsis protoplasts (Li et al., 2015).

We also tested and compared the editing efficiency with plasmid DNA or RNP using dual gRNAs targeting AtPDS (AT4G14210) and AtFAD2 (AT3G12120, referred to as AtFAD below in the text) in Arabidopsis protoplasts. Three independent biological experiments with triplicate samples for each independent experiment were conducted to determine the editing efficiency mediated by NHEJ. NGS analyses revealed a significantly higher RNP editing efficiency at $61.3 \% \pm 5.1$ and $62.6 \% \pm 1.7$ in AtPDS and AtFAD, respectively, than those of DNA at $5.9 \% \pm 3.4$ and $12.1 \% \pm 1.9$, respectively (Supplementary Figures S2, S3). The results suggested that the preassembled RNPs could be more efficient in genome editing of three independent Arabidopsis genes using protoplast assays.

To investigate whether Cas9 RNP could induce undesired edits at the genome level in Arabidopsis protoplasts, CasOFFinder was used to predict off-target sites for the gRNAs targeting AtBON1. We did not find any off-targets by PCR and sequencing analyses of the predicted off-target sites for AtBON1 gRNAs (data not shown).

\section{Cas9-RNP Activity in Editing NHEJ-GFP and HDR-GFP Reporter Genes}

Highly efficient and rapid transient expression analyses in mesophyll protoplasts provide an excellent platform to rapidly evaluate gene editing efficiency of emerging Cas9-gRNA variants (Yoo et al., 2007; Li et al., 2013, 2014; Lin et al., 2020; Lin et al., 2021; Zhang et al., 2021). To further simplify and lower the cost for sensitive detection of gene editing mediated via NHEJ or HDR by Cas 9 complexes, we developed two GFP reporter genes, NHEJGFP (Figure 3A ) and HDR-GFP (Figure 3B ), that can be easily applied in a broad range of established or new protoplast systems for diverse plant species. For example, gene editing by Cas9gRNA variants could generate a double-strand break and inframe shift via NHEJ or the precise 65TLR67 to 65TYG67 mutation by HDR with a ssODN donor, leading to the recovery of GFP fluorescence. UBQ10-NLS-tdTomato was cotransfected and served as a control to determine the transfection efficiency (Figure 3C). Quantitative analyses determined by the ratio of GFP/tdTomato in Arabidopsis protoplasts demonstrated a significantly higher gene editing efficiency by Cas9 RNP (53.3\%) than by DNA transfection to express Cas9-gRNA (29.6\%) (Figure 3D). To test GFP reporter recovery by NHEJmediated repair in different plant species, protoplasts isolated from $N$. benthamiana, $A$. thaliana, $B$. rapa and $C$. sativa (Figure 1A) were co-transfected with Cas9 RNP and NHJEGFP (Figure 3A). The recovered GFP signal mediated by Cas9-RNP was detected in the nuclei of the transfected protoplasts of $N$. benthamiana, A. thaliana, B. rapa and $C$. sativa at the editing efficiency of $85,56,63$ and $26 \%$, respectively (Figure $\mathbf{3 F}$ ).

To detect precise gene editing via HDR, we co-transfected ssODN complementary to the non-target strand as the DNA donor and HDR-GFP (Figure 3B) with preassembled Cas9 RNP. We first determined the efficacy of the length of individual ssODN for HDR-mediated GFP recovery in N. benthamiana protoplasts, which appeared to exhibit the highest efficiency in genome editing from previous studies (Li et al., 2013). Longer ssODN up to $70 \mathrm{nt}$ as the donor template for HDR promoted the repair of GFP at the highest efficiency of $45 \%$. However, even a 36-nt short symmetric ssODN complementary to the non-target strand produced sufficiently high HDR-mediated gene editing (Figure 3E) (Richardson et al., 2016). The same $70 \mathrm{nt}$ ssODN template was further tested for the efficiency of HDR-directed mutant GFP recovery in the protoplasts isolated from $N$. benthamiana, A. thaliana, B. rapa and $C$. sativa and transfected with Cas9 RNP. Recovered GFP signal was detected in 50,30, 33 and $19 \%$ of the transfected protoplasts of N. benthamiana, A. thaliana, B. rapa and C. sativa, respectively (Figure 3F). The findings suggested that the sensitive GFP reporter genes will be valuable and facile tools for the development of CRISPR technologies and for advancing our understanding of the key molecular mechanisms in plants.

\section{Cas9-RNP-ssODN Mediates HDR in the Genome}

The efficacy of Cas9-RNP/ssODN for HDR-mediated precise gene editing was further tested in the genome. AtALS (AT3G48560) is an ideal gene for the HDR efficiency testing in plants because change of only one amino acid at the defined site confers herbicide resistance (Haughn, et al., 1988). To enhance the efficiency, we generated two neighboring gRNAs on the opposite strand (Li et al., 2015) and synthesized the ssODN to mediate the specific Pro/CCT to Ser/TCA mutation with or without Cas9 RNPs (Figure 4A). The results showed 7.2 and 4.0\% HDR-mediated genomic editing efficiency at the target site in two independent biological experiments, whereas no HDR was 
A

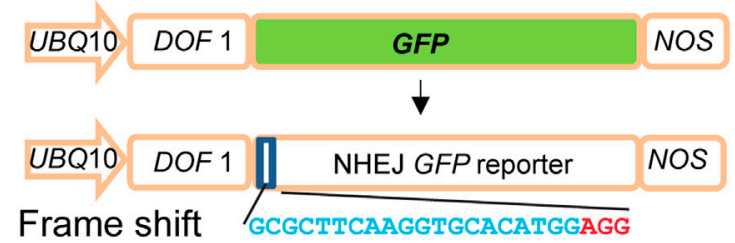

Frame shift GCGCTTCAAGGTGCACATGGAGG

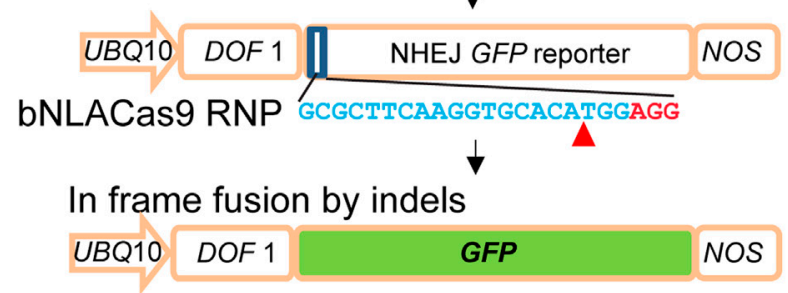

B

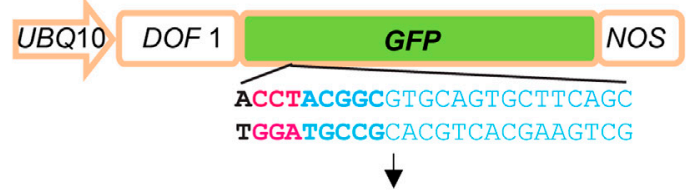

ACC TAC GGC to ACC TTA CGC (65TYG67 to TLR)

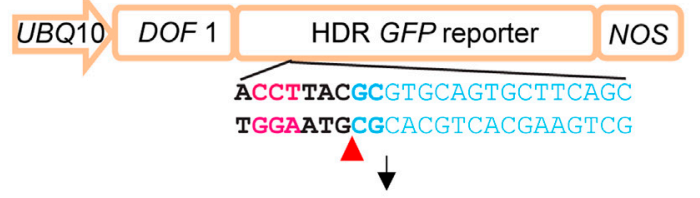

ACC TTA CGC to ACC TAC GGC (65TLR67 to TYG) bNLSCas9 RNP + ssODN (70 nt) ---ACCTACGGCGTG-

\begin{tabular}{llll}
\hline UBQ10 & DOF 1 & GFP & NOS
\end{tabular}
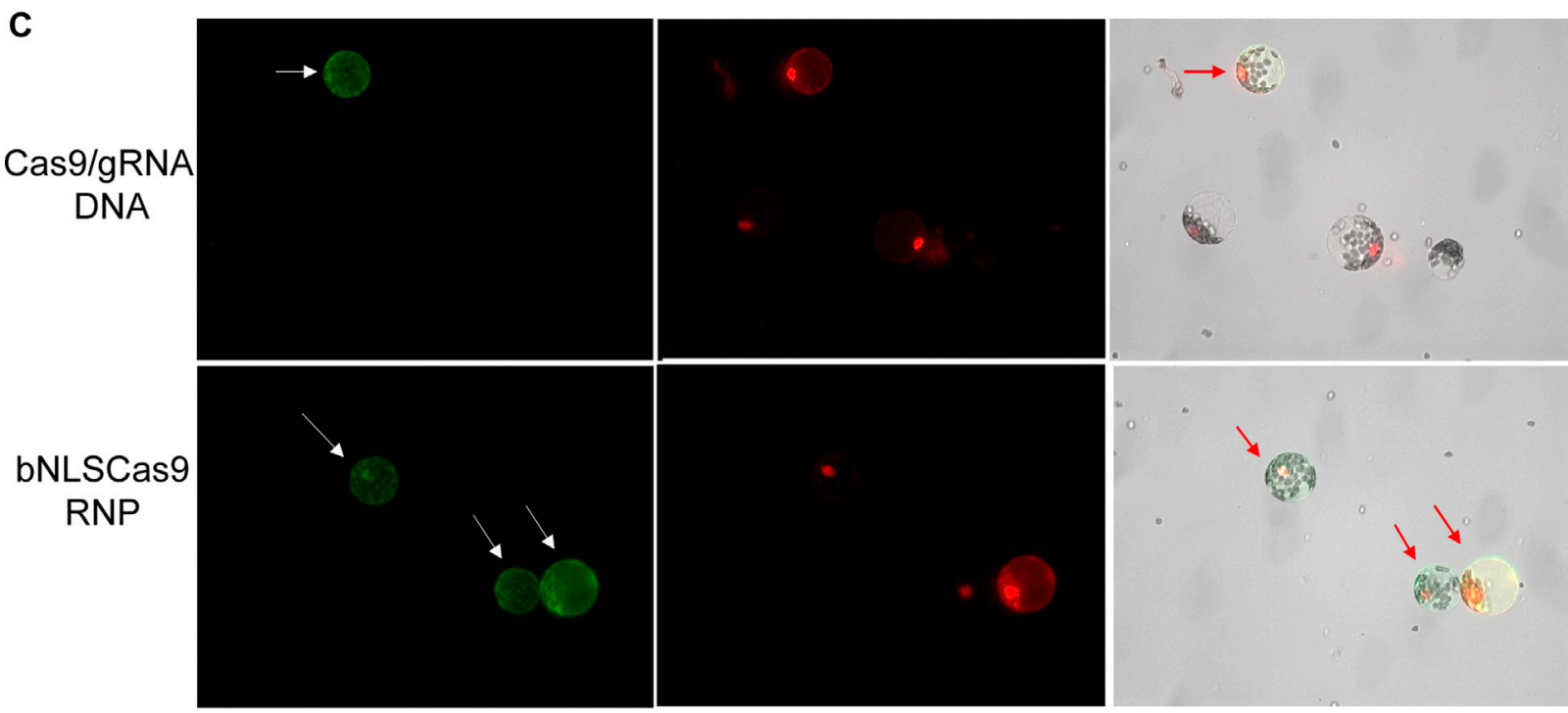

D

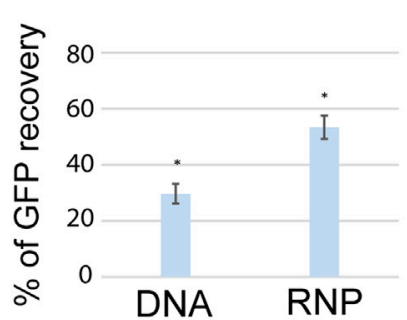

E

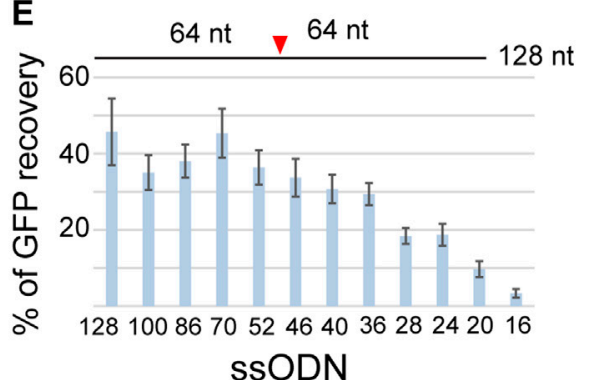

$\mathbf{F}$

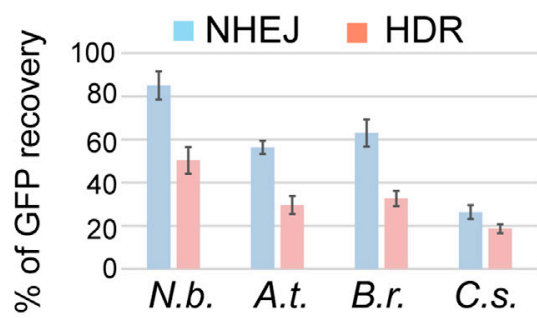

FIGURE 3 | Facile GFP reporter genes for sensitive NHEJ and HDR detection in protoplasts. (A) The design and analysis of the NHEJ GFP reporter gene. (B) The design and analysis of the HDR GFP reporter gene. (C) Comparison of GFP recovery efficiency by NHEJ via DNA transfection or bNLSCas9 RNP in Arabidopsis protoplasts. Protoplasts were co-transfected with UBQ10-tdTomato for normalization. (D) Quantitative analysis of GFP recovery by DNA- or RNP-mediated gene editing. (E) The length of ssODN donor determines HDR editing efficiency by bNLSCas9 RNP in tobacco protoplasts. (F) Efficient editing of GFP reporter genes in four protoplast systems. Blue bar, NHEJ. Red bar, HDR. SD is shown $(n=3), p<0.001$. The sequences of gRNAs are shown with the PAM sequence in red. Red arrowhead indicates the double-strand break site in the GFP reporter gene. UBQ10, the A.t. UBIQUITIN10 gene promoter. DOF1, the nuclear localization signal. NOS, the NOS gene terminator. At, A. thaliana, N.b., N. benthamiana, B. r., B. rapa, and C.s., C. sativa. 
A Editing AtALS (AT3G48560) by HDR bNLSCas9 RNP (gRNA1 + gRNA2)

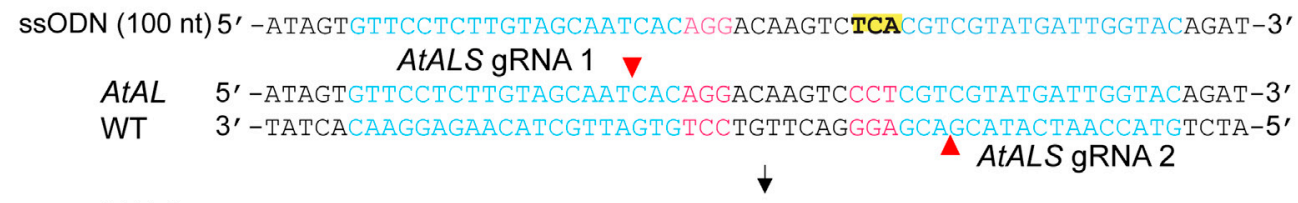

AtAlS 5'-AtAgtgTtCCTCTtGTAGCAATCACAGGACAAGTCTCACGTCGTATGATTGGTACAGAT-3'

Edited 3'-TATCACAAGGAGAACATCGTTAGTGTCCTGTTCAGAGTGCAGCATACTAACCATGTCTA-5'

B

\begin{tabular}{|c|c|c|}
\hline & AtALS gRNA 2 & \\
\hline $\begin{array}{c}\text { Exp. } 1 \\
\text { bNLA Cas9 RNP } \\
\text { ssODN } 7.2 \%\end{array}$ & 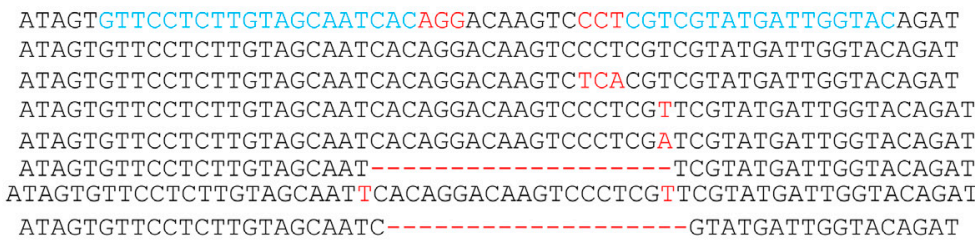 & $\begin{array}{l}\text { WT } \\
86.8 \% \\
7.2 \% \\
0.99 \% \\
0.91 \% \\
0.63 \% \\
0.51 \% \\
0.49 \%\end{array}$ \\
\hline $\begin{array}{c}\text { Exp.2 } \\
\text { bNLSCas9 RNP } \\
\text { ssODN } \\
4.0 \%\end{array}$ & $\begin{array}{l}\text { ATAGTGTTCCTCTTGTAGCAATCACAGGACAAGTCCCTCGTCGTATGATTGGTACAGAT } \\
\text { ATAGTGTTCCTCTTGTAGCAATCACAGGACAAGTCCCTCGTCGTATGATTGGTACAGAT } \\
\text { ATAGTGTTCCTCTTGTAGCAATCACAGGACAAGTCTCACGTCGTATGATTGGTACAGAT } \\
\text { ATAGTNGTTCCTCTTGTAGCAATC------------GTATGATTGGTACAGAT } \\
\text { ATAGTGTTCCTCTTGTAGCAATCACAGGACAAGTCCCTCGATCGTATGATTGGTACAGAT } \\
\text { ATAGTGTTCCTCTTGTAGCAATCACAGGACAAGTCCCTCGTTCGTATGATTGGTACAGAT } \\
\text { ATAGTGTTCCTCTTGTAGCAAT-------------TCGTATGATTGGTACAGAT } \\
\text { ATAGTGTTCCTCTTGTAGCAATCACAGGACAAGTCCCTCG-CGTATGATTGGTACAGAT } \\
\text { ATAGTGTTCCTCTTGTAGCAATCACAGGACAAGTCCCTCGT---ATGATGGTACAGAT }\end{array}$ & $\begin{array}{l}\text { WT } \\
83.5 \% \\
4.0 \% \\
1.7 \% \\
1.3 \% \\
1.0 \% \\
0.97 \% \\
0.94 \% \\
0.76 \%\end{array}$ \\
\hline
\end{tabular}

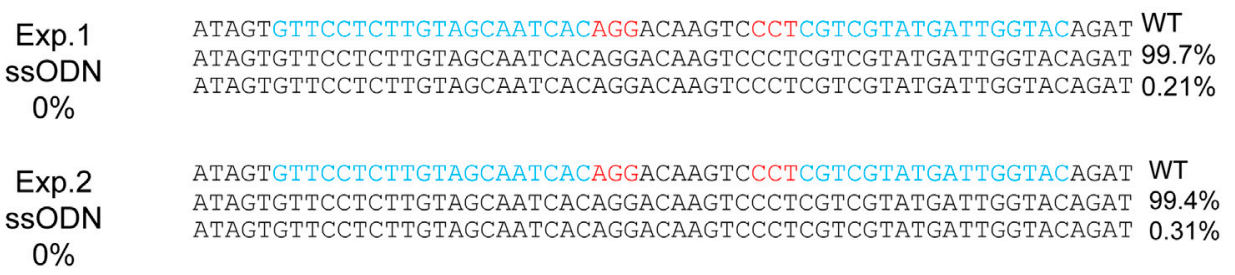

FIGURE 4 | Precise bNLSCas9 RNP-mediated mutagenesis. (A) The experimental design for precise mutagenesis via HDR by bNLSCas9 RNP and ssODN. (B) Specific AtALS mutation generated by bNLSCas9 RNP and SSODN in Arabidopsis protoplasts. Protoplasts were transfected with SsODN without RNP served as the background control. Editing efficiency (red \%) was shown by NGS analyses of the amplicons generated by PCR using genomic DNA isolated from transfected protoplasts.

detected when Cas9 RNPs was absent (Figure 4B). The findings paved the way for generating precisely targeted genomic changes in plants regenerated from engineered protoplasts (Lin et al., 2018; Hsu et al., 2021; Zhang et al., 2021).

\section{PE RNPs Mediate Precise Prime Editing}

The emerging PEs for versatile genome editing without doublestrand breaks and donor DNA were derived from a protein fusion with Cas9 nickase (H840A) and the mammalian viral M-MLV RTase (nCas9-RT). PE3b was most active in human tumor culture cells (HEK293T), but its requirement for a second gRNA-mediated nick appeared to enhance unintended indels in mouse embryos (Anzalone et al., 2019; Aida et al., 2020; Lin et al., 2021). We expressed an nCas9-RT derived from PE2 and bNLSCas9 in E. coli for the PE-RNP-mediated gene editing assays using HDR-GFP in N. benthamiana protoplasts (Figure 5A). For optimizing PE-RNP-mediated HDR efficiency, primer binding site (PBS), which was kept at $13 \mathrm{nt}$, and the RT template (RTT) were tested from 10 to $34 \mathrm{nt}$ in pegRNAs (Figure 5B). GFP recovery in transfected $N$. benthamiana protoplasts showed that the ideal RTT length was between $16-18 \mathrm{nt}$ to reach $50 \%$ efficiency in precise gene editing (Figure 5C). We also tested the efficacy of RT RNPs with a specific pegRNA in precisely editing the AtPDS gene in the genome. The change from $\mathrm{C}$ to $\mathrm{T}$ introduced a stop codon TAG in the AtPDS coding region. NGS analyses of the amplicons detected up to $4.6 \%$ precise base replacement caused by PE RNPs (Figure 5D). The off-target frequency caused by PE RNP was very low at $0-0.02 \%$ in Arabidopsis protoplasts (Supplementary Figure S4). This finding is consistent with the result reported for PE-mediated genome editing 


\section{A Precise nCas9-RT RNP-mediated mutagenesis}

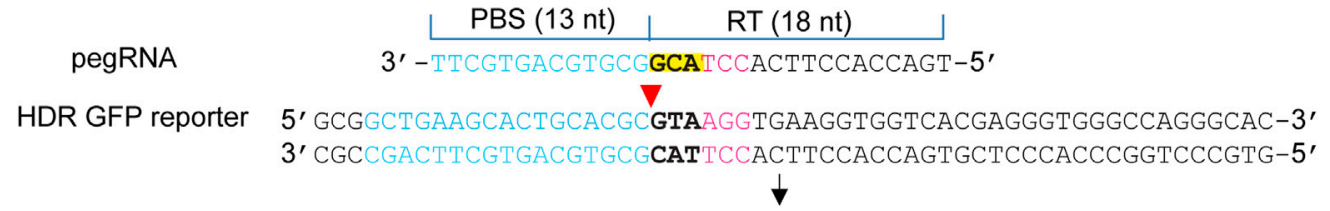

Edited GFP

5' GCGGCTGAAGCACTGCACGCCGTAGGTGAAGGTGGTCACGAGGGTGGGCCAGGGCAC-3' 3' CGCCGACTTCGTGACGTGCGGCATCCACTTCCACCAGTGCTCCCACCCGGTCCCGTG-5'

B

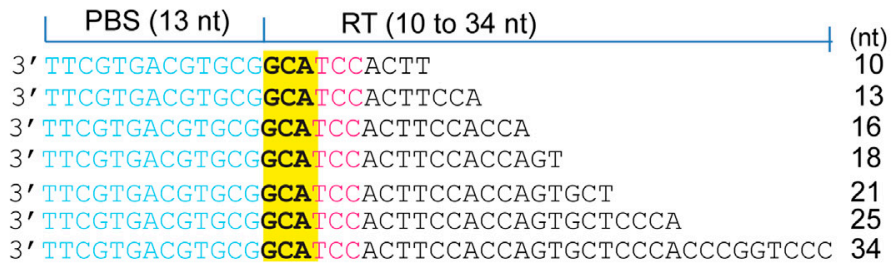

C

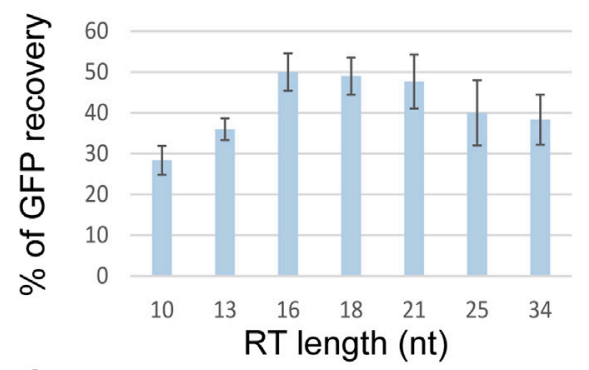

D nCas9-RT RNP mutagenesis

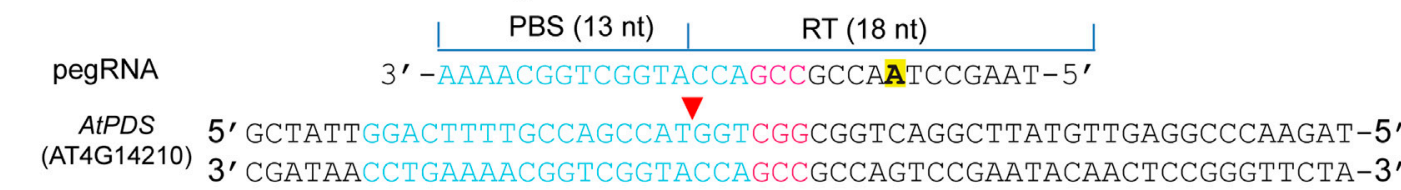

E

Exp. 1
$4.6 \%$

GCTATTGGACTTTTGCCAGCCATGGTCGGCGGTCAGGCTTATGTTGAGGCCCAAGAT WT GCTATTGGACTTTTGCCAGCCATGGTCGGCGGTCAGGCTTATGTTGAGGCCCAAGAT $94.5 \%$ GCTATTGGACTTTTGCCAGCCATGGTCGGCGGTTAGGCTTATGTTGAGGCCCAAGAT $4.6 \%$ GCTATTGGACTTTTGCCAGCCATGGTCGGCGGTCAGGCTTATGTTGAGGCCCAAGAT $\quad 0.48 \%$ GCTATTGGACTTTTGCCAGCCATGGTCGGCGGTCAGGCTTATGTTGAGGCCCAAGAT $\quad 0.22 \%$

Exp.2 GCTATTGGACTTTTGCCAGCCATGGTCGGCGGTCAGGCTTATGTTGAGGCCCAAGAT WT $1.8 \%$ GCTATTGGACTTTTGCCAGCCATGGTCGGCGGTCAGGCTTATGTTGAGGCCCAAGAT 97.6\% GCTATTGGACTTTTGCCAGCCATGGTCGGCGGTTAGGCTTATGTTGAGGCCCAAGAT $\quad 1.8 \%$ GCTATTGGACTTTTGCCAGCCATGGTCGGCGGTCAGGCTTATGTTGAGGCCCAAGAT $0.62 \%$

FIGURE 5 | Precise PE RNP-mediated mutagenesis. (A) The experimental design for precise mutagenesis by PE RNP. (B) Analyses of pegRNA variants. (C) RT template length affects PE RNP-mediated editing in the HDR GFP reporter gene. (D) The experimental design to generate a precise AtPDS mutation by PE RNPmediated editing in the genome. (E) A specific AtPDS mutation generated by PE RNP in Arabidopsis protoplasts. Editing efficiency (red \%) was shown by NGS analyses of the amplicons generated by PCR using genomic DNA isolated from transfected protoplasts.

in rice protoplasts with off-target frequency at $0.00-0.23 \%$ (Jin et al., 2021). The rapid tests with remarkably high efficiency and RTT length optimization based on the HDR-GFP reporter gene provided insightful information to support the experimental design for successful exploration of new PE RNPs not previously validated at the genomic level in any organisms.

\section{DISCUSSION}

By combining multiple advantages of the superior flexibility and high efficacy of Cas9 RNPs with rapid and versatile transient assays, our studies demonstrated that the plant protoplast platform is simple, robust, economical and generally applicable 
in multiple plant species to support the rapidly evolving advances and innovations of CRISPR technologies. Easy guidelines and universal principles are provided for the selection of optimal leaves as the sources of high-quality protoplasts with desirable transfection efficiency in a wide range of model and crop plants. Unlike the commonly used mammalian cell lines, convenient seed storage, germination and easy plant care provide the most reliable and abundant primary plant materials without cell culture maintenance. Moreover, cell cycle could be manipulated in mesophyll protoplasts (Xiong et al., 2013) to potentially enhance precision genome editing via HDR with short ssODN by Cas9 RNPs or PE RNPs without double-strand break and donor DNA. The protoplast systems integrating Cas9 RNPs or PE RNPs with highly efficient protoplast transfection using sensitive and versatile GFP reporter genes enable rapid screens and analyses of NHEJ-, HDR- or RT-mediated gene editing efficiency. The platform is most suitable for comprehensive testing and systematic evaluation of various Cas9gRNA tools and new designs for multiplex and precision genome editing, as well as for further research to elucidate the underlying molecular mechanisms.

We fully recognize that the production and purification of high-quality Cas proteins and synthetic gRNAs and pegRNAs to achieve high genome editing efficiency in protoplasts is still a significant challenge for many plant labs (Zhang et al., 2021). Our intention is to share our accumulated experiences with promising results using RNPs in four different eudicot protoplast systems. We hope that the new positive findings may encourage others to try RNPs in their favorite plant systems to potentially obtain higher genome editing efficiency not previously possible using plasmid DNA. For successful genome editing experiments in protoplasts, paying attention to every detail (MATERIALS and MATHODS; Yoo et al., 2007; Li et al., 2013, 2015) from plant growth condition, leaf age and selection, protoplast isolation and transfection, to RNP production and assembly, gRNA design and quality, and NLS-tag are all crucial in the development of highly efficient protoplast systems with different plant species.

Current applications of CRISPR technologies in basic plant and agricultural research are mostly limited to the error-prone NHEJ-generated mutants using Agrobacterium- or particle bombardment-mediated plant transformation, often yielding unpredictable outcomes or traits. The protoplast platform will facilitate the development of precision genome editing at high efficiency using Cas9 RNPs or PE RNPs without transgenes or selection markers. The platform is a prerequisite for ongoing efforts to advance genome edited protoplasts to regenerated

\section{REFERENCES}

Aida, T., Wilde, J. J., Yang, L., Hou, Y., Li, M., Xu, D., et al. (2020). Prime Editing Primarily Induces Undesired Outcomes in Mice. bioRxiv. doi:10.1101/ 2020.08.06.239723

Anzalone, A. V., Randolph, P. B., Davis, J. R., Sousa, A. A., Koblan, L. W., Levy, J. M., et al. (2019). Search-and-replace Genome Editing without Double-Strand Breaks or Donor DNA. Nature 576, 149-157. doi:10.1038/s41586-019-1711-4

Chechik, L., Martin, O., and Soutoglou, E. (2020). Genome Editing Fidelity in the Context of DNA Sequence and Chromatin Structure. Front. Cel Dev. Biol. 8, 319. doi:10.3389/fcell.2020.00319 plants without genome instability (Woo et al., 2015; Lin et al., 2018; Hsu et al., 2021; Zhang et al., 2021). Although plant regeneration can be very difficult in some plants, with the development of protoplast methodology for plant regeneration of various species, the protoplast platform has broad application prospects. Future advances in the development of new delivery strategies, such as the promising and comprehensive nanoparticle technologies (Kumar et al., 2020; Lv et al., 2020) for introducing Cas9 RNPs or PE RNPs directly into pollen, zygotes, embryos and regenerating meristems (Toda et al., 2019; Zhang et al., 2021), will offer powerful alternatives for versatile and precise genetic engineering in diverse plants.

\section{DATA AVAILABILITY STATEMENT}

The original contributions presented in the study are included in the article/Supplementary Materials, further inquiries can be directed to the corresponding author.

\section{AUTHOR CONTRIBUTIONS}

WJ and JS conceived and initiated the project, WJ performed experiments, JB helped growing plants, JS and WJ wrote the manuscript. All authors discussed the results and commented on the manuscript.

\section{FUNDING}

This work was supported by the NIH grants GM060493 and GM129093.

\section{ACKNOWLEDGMENTS}

We thank CS. Lin for insightful discussions.

\section{SUPPLEMENTARY MATERIAL}

The Supplementary Material for this article can be found online at: https://www.frontiersin.org/articles/10.3389/fgeed.2021.719190/ full\#supplementary-material

Chiu, W.-l., Niwa, Y., Zeng, W., Hirano, T., Kobayashi, H., and Sheen, J. (1996). Engineered GFP as a Vital Reporter in Plants. Curr. Biol. 6, 325-330. doi:10.1016/s0960-9822(02)00483-9

D’Astolfo, D. S., Pagliero, R. J., Pras, A., Karthaus, W. R., Clevers, H., Prasad, V., et al. (2015). Efficient Intracellular Delivery of Native Proteins. Cell 161, 674-690. doi:10.1016/j.cell.2015.03.028

Emonet, A., Zhou, F., Vacheron, J., Heiman, C. M., Dénervaud Tendon, V., Ma, K.W., et al. (2021). Spatially Restricted Immune Responses Are Required for Maintaining Root Meristematic Activity upon Detection of Bacteria. Curr. Biol. 31, 1012-1028. doi:10.1016/j.cub.2020.12.048

Haughn, G. W., Smith, J., Mazur, B., and Somerville, C. (1988). Transformation with a Mutant Arabidopsis Acetolactate Synthase Gene Renders Tobacco 
Resistant to Sulfonylurea Herbicides. Mol. Gen. Genet. 211, 266-271. doi:10.1007/bf00330603

Hsu, C.-T., Yuan, Y.-H., Lin, Y.-C., Lin, S., Cheng, Q.-W., Wu, F.-H., et al. (2021). Efficient and Economical Targeted Insertion in Plant Genomes via Protoplast Regeneration. CRISPR J. 4, 752-760. doi:10.1089/crispr.2021.0045

Jin, S., Lin, Q., Luo, Y., Zhu, Z., Liu, G., Li, Y., et al. (2021). Genome-wide Specificity of Prime Editors in Plants. Nat. Biotechnol. 39, 1292-1299. doi:10.1038/s41587-02100891-x

Kumar, S., Nehra, M., Dilbaghi, N., Marrazza, G., Tuteja, S. K., and Kim, K.-H. (2020). Nanovehicles for Plant Modifications towards Pest- and Disease-Resistance Traits. Trends Plant Sci. 25, 198-212. doi:10.1016/j.tplants.2019.10.007

Li, J.-F., Norville, J. E., Aach, J., McCormack, M., Zhang, D., Bush, J., et al. (2013). Multiplex and Homologous Recombination-Mediated Genome Editing in Arabidopsis and Nicotiana Benthamiana Using Guide RNA and Cas9. Nat. Biotechnol. 31, 688-691. doi:10.1038/nbt.2654

Li, J.-F., Zhang, D., and Sheen, J. (2014). Epitope-tagged Protein-Based Artificial miRNA Screens for Optimized Gene Silencing in Plants. Nat. Protoc. 9, 939-949. doi:10.1038/nprot.2014.061

Li, J.-F., Zhang, D., and Sheen, J. (2015). Targeted Plant Genome Editing via the CRISPR/Cas9 Technology. Methods Mol. Biol. 1284, 239-255. doi:10.1007/9781-4939-2444-8_12

Lin, C.-S., Hsu, C.-T., Yang, L.-H., Lee, L.-Y., Fu, J.-Y., Cheng, Q.-W., et al. (2018). Application of Protoplast Technology to CRISPR/Cas9 Mutagenesis: from Single-Cell Mutation Detection to Mutant Plant Regeneration. Plant Biotechnol. J. 16, 1295-1310. doi:10.1111/pbi.12870

Lin, Q., Zong, Y., Xue, C., Wang, S., Jin, S., Zhu, Z., et al. (2020). Prime Genome Editing in rice and Wheat. Nat. Biotechnol. 38, 582-585. doi:10.1038/s41587-020-0455-x

Lin, Q., Jin, S., Zong, Y., Yu, H., Zhu, Z., Liu, G., et al. (2021). High-Efficiency Prime Editing with Optimized, Paired pegRNAs in Plants. Nat. Biotechnol. 39, 923-927. doi:10.1038/s41587-021-00868-w

Lu, Y., Tian, Y., Shen, R., Yao, Q., Wang, M., Chen, M., et al. (2020). Targeted, Efficient Sequence Insertion and Replacement in rice. Nat. Biotechnol. 38, 1402-1407. doi:10.1038/s41587-020-0581-5

Lv, Z., Jiang, R., Chen, J., and Chen, W. (2020). Nanoparticle-Mediated Gene Transformation Strategies for Plant Genetic Engineering. Plant J. 104, 880-891. doi:10.1111/tpj.14973

Marx, V. (2016). Plants: a Tool Box of Cell-Based Assays. Nat. Methods 13, 551-554. doi:10.1038/nmeth.3900

Richardson, C. D., Ray, G. J., DeWitt, M. A., Curie, G. L., and Corn, J. E. (2016). Enhancing Homology-Directed Genome Editing by Catalytically Active and Inactive CRISPR-Cas9 Using Asymmetric Donor DNA. Nat. Biotechnol. 34, 339-344. doi:10.1038/nbt.3481
Shan, Q., Wang, Y., Li, J., Zhang, Y., Chen, K., Liang, Z., et al. (2013). Targeted Genome Modification of Crop Plants Using a CRISPR-Cas System. Nat. Biotechnol. 31, 686-688. doi:10.1038/nbt.2650

Toda, E., Koiso, N., Takebayashi, A., Ichikawa, M., Kiba, T., Osakabe, K., et al. (2019). An Efficient DNA- and Selectable-Marker-Free Genome-Editing System Using Zygotes in rice. Nat. Plants 5, 363-368. doi:10.1038/s41477019-0386-z

Woo, J. W., Kim, J., Kwon, S. I., Corvalán, C., Cho, S. W., Kim, H., et al. (2015). DNA-free Genome Editing in Plants with Preassembled CRISPR-Cas9 Ribonucleoproteins. Nat. Biotechnol. 33, 1162-1164. doi:10.1038/nbt.3389

Xiong, Y., McCormack, M., Li, L., Hall, Q., Xiang, C., and Sheen, J. (2013). GlucoseTOR Signalling Reprograms the Transcriptome and Activates Meristems. Nature 496, 181-186. doi:10.1038/nature12030

Yanagisawa, S., and Sheen, J. (1998). Involvement of Maize Dof Zinc Finger Proteins in Tissue-specific and Light-Regulated Gene Expression. Plant Cel. 10, 75-89. doi:10.1105/tpc.10.1.75

Yoo, S.-D., Cho, Y.-H., and Sheen, J. (2007). Arabidopsis Mesophyll Protoplasts: A Versatile Cell System for Transient Gene Expression Analysis. Nat. Protoc. 2, 1565-1572. doi:10.1038/nprot.2007.199

Zhang, Y., Iaffaldano, B., and Qi, Y. (2021). CRISPR Ribonucleoprotein-Mediated Genetic Engineering in Plants. Plant Commun. 2, 100168. doi:10.1016/ j.xplc.2021.100168

Zhu, H., Li, C., and Gao, C. (2020). Applications of CRISPR-Cas in Agriculture and Plant Biotechnology. Nat. Rev. Mol. Cel Biol. 21, 661-677. doi:10.1038/s41580020-00288-9

Conflict of Interest: The authors declare that the research was conducted in the absence of any commercial or financial relationships that could be construed as a potential conflict of interest.

Publisher's Note: All claims expressed in this article are solely those of the authors and do not necessarily represent those of their affiliated organizations, or those of the publisher, the editors and the reviewers. Any product that may be evaluated in this article, or claim that may be made by its manufacturer, is not guaranteed or endorsed by the publisher.

Copyright $\odot 2021$ Jiang, Bush and Sheen. This is an open-access article distributed under the terms of the Creative Commons Attribution License (CC BY). The use, distribution or reproduction in other forums is permitted, provided the original author(s) and the copyright owner(s) are credited and that the original publication in this journal is cited, in accordance with accepted academic practice. No use, distribution or reproduction is permitted which does not comply with these terms. 